\title{
Signatures of polaronic charge ordering in optical and de conductivity using dynamical mean field theory
}

\author{
S. Ciuchi \\ Dipartimento di Fisica, Università dell'Aquila and SMC Research Center INFM-CNR, CNISM, via Vetoio, \\ I-67010 Coppito-L'Aquila, Italy \\ S. Fratini* \\ Institut Néel, CNRS and Université Joseph Fourier, BP 166, F-38042 Grenoble Cedex 9, France
}

(Received 28 February 2008; published 30 May 2008)

\begin{abstract}
We apply dynamical mean field theory to study a prototypical model that describes charge ordering in the presence of both electron-lattice interactions and intersite electrostatic repulsion between electrons. We calculate the optical and dc conductivity and derive approximate formulas valid in the limiting electron-lattice coupling regimes. In the weak-coupling regime, we recover the usual behavior of charge density waves, characterized by a transfer of spectral weight due to the opening of a gap in the excitation spectrum. In the opposite limit of very strong electron-lattice coupling, instead, the charge ordering transition is signaled by a global enhancement of the optical absorption, with no appreciable spectral weight transfer. Such behavior is related to the progressive suppression of thermally activated charge defects taking place below the critical temperature. At intermediate values of the coupling within the polaronic regime, a complex behavior is obtained where both mechanisms of transfer and enhancement of spectral weight coexist.
\end{abstract}

DOI: 10.1103/PhysRevB.77.205127

PACS number(s): 71.38.-k, 78.20.Bh, 71.45.Lr

\section{INTRODUCTION}

As opposed to conventional charge density waves, ${ }^{1}$ which are well understood in terms of lattice-driven instabilities of the Fermi surface in metallic systems, there is no unified description of the charge ordering ( $\mathrm{CO}$ ) transitions observed in strongly interacting systems or "bad metals." Typical examples of such systems are transition-metal oxides, which are invariably characterized by a complex interplay between several microscopic interactions, involving the charge, spin, orbital, and lattice degrees of freedom. This complexity seems to preclude the identification of a simple, common, charge ordering mechanism. Still, electron-lattice interactions are always present to some extent and often play a dominant role in driving the $\mathrm{CO}$ transition in these systems. Charge ordering phenomena involving a strong electronlattice coupling have been found in manganites, ${ }^{2-4}$ nickelates, ${ }^{5,6}$ layered cobaltates, ${ }^{7,8}$ magnetite, ${ }^{9-11}$ vanadates, ${ }^{12,13}$ and oxoborates, ${ }^{14}$ as well as in other inorganic low-dimensional systems. ${ }^{15,16}$ The lattice degrees of freedom could also be relevant in the charge ordered phases of the so-called "telephone-number" ladder compounds, ${ }^{17}$ as well as in two-dimensional organic salts, ${ }^{18}$ although in those cases, most theoretical interpretations up to now have focused on models with purely electronic interactions.

In this work, we focus on a minimal model that describes charge ordering in the presence of electron-lattice interactions, with particular attention to the polaronic regime obtained at strong coupling. The model consists of electrons on a bipartite lattice at a commensurate concentration of one electron on every two sites, interacting locally with dispersionless lattice vibrations. The electrons also interact mutually via an intersite electrostatic repulsion, which can be thought of as the screened part of the long-ranged Coulomb potential. While this oversimplified model only retains part of the complex physics involved in real systems, its solution can be helpful to clarify certain aspects of the charge ordering phenomena that are common to systems with strong electron-lattice interactions and that can, in principle, be identified experimentally. To be specific, we solve the model by performing the following approximations.

(i) The magnetic degrees of freedom are taken out of the game by resorting to spinless electrons. This enforces locally the constraint of no double occupancy characteristic of the limit of strong "Hubbard" (on-site) repulsion. It is appropriate for our purposes as long as we do not aim at describing the effects of electronic correlations on the low-energy physics, such as the existence of a quasiparticle peak or the induced magnetic exchange. In principle, this approximation is viable as long as the magnetic and charge degrees of freedom are governed by different energy scales. In such case, the magnetic interactions are not expected to have a strong influence on the charge ordering pattern (although exceptions do exist $\left.{ }^{19-21}\right)$. This separation of energy scales is realized, for instance, in magnetite $\left(\mathrm{Fe}_{3} \mathrm{O}_{4}\right),{ }^{22}$ where the $\mathrm{CO}$ transition occurs within a ferromagnetic phase that preexists at a much higher temperature. More examples can be found in systems [such as $\mathrm{V}_{3} \mathrm{O}_{5}$ (Ref. 13)] where the $\mathrm{CO}$ transition takes place within a paramagnetic phase, the magnetic order setting in at a much lower temperature. The spinless approximation is also relevant to half-metals, such as the colossal magnetoresistance manganites.

(ii) We consider a single electronic band, which rules out the orbital degrees of freedom and their possible ordering, which is known to play an important role in specific compounds.

(iii) The lattice vibrations are assumed adiabatic, i.e., their characteristic energy is much smaller than the electronic bandwidth, which is typically the case in transition-metal oxides. The adiabatic approximation is enforced here by 
treating the lattice degrees of freedom as static variables with a given (thermal) statistical distribution. As a consequence, the low-energy spectral features that derive from the quantum nature of the phonons are lost. These would appear at energies comparable to or below the phonon energies. Such features are anyhow of minor quantitative importance in the polaronic regime of interest here, where most of the spectral weight is moved to higher energies, of the order of the polaron binding energy. The same argument justifies the neglect of low-energy phenomena originating from electronic correlations, as discussed at point (i) above, in all cases where the polarons set the dominant energy scale.

(iv) We apply single-site dynamical mean field theory (DMFT), appropriately adapted ${ }^{19,23-25}$ to account for the charge unbalance between neighboring sites in the charge ordered state. This approach is known to deal very effectively with local interaction mechanisms, regardless of their strength. It is therefore expected to give an accurate description of the electron-lattice physics. As was shown in Ref. 23, this approach correctly describes the crossover between the weak-coupling charge density wave regime and strongcoupling polaronic regime and allows us to shed light on the role played by defects of the lattice polarization at the ordering transition. A lesser accuracy is achieved in treating the intersite Coulomb repulsion since nonlocal interactions reduce to the mean field (Hartree) level in single-site DMFT. This is, however, not a primary limitation ${ }^{19,20}$ in the regime we are mainly interested in, where the physics is dominated by the electron-lattice interaction.

The main result of this work is the identification of qualitatively different behaviors of the electrodynamic response in the two fundamentally distinct regimes of charge ordering, governed by the strength of the electron-lattice interaction. In the weak-coupling regime, the electrodynamic response is dominated by the opening of an energy gap in the excitation spectrum of an otherwise metallic system. At the ordering transition, the optical spectral weight is transferred from the Drude peak to the region above the gap, and the dc conductivity acquires a semiconducting character. On the contrary, in the strong-coupling limit, charge localization is already present in the normal phase due to the formation of selflocalized polarons. Correspondingly, the low-energy spectrum is already strongly depleted by a polaronic "pseudogap," and the genuine gap opening occurring at the transition does not give rise to any appreciable depletion or shift of the low-frequency spectral weight. Instead, the ordering of polarons results in a global enhancement of the optical spectral weight, which is opposite to what is expected from a conventional charge density wave. The two scenarios described above coexist in the polaronic regime at moderate values of the electron-lattice coupling. This leads to a complex behavior presenting both a transfer and an enhancement of the spectral weight, resulting in a nonmonotonic temperature dependence of the total optical weight within the $\mathrm{CO}$ phase. The inclusion of a direct intersite Coulomb interaction extends this complex intermediate region to larger electronlattice interaction strengths.

The solution for the one-particle spectral function has already been published in Ref. 23 in a model with electron- lattice interactions alone. Here, we extend that treatment to calculate the optical and dc conductivity throughout the parameter space, including an intersite Coulomb repulsion between electrons. We also derive analytical expressions for these quantities that are valid in the polaronic regime, elucidating the role played by charge defects in both optical absorption and charge transports. It can be noted that an analogous model, without the Coulomb interaction term, was studied variationally in Ref. 26 . There, however, the physics of defects was not addressed.

The paper is organized as follows. The general formalism for treating the charge ordered broken-symmetry phase within the DMFT is presented in Sec. II. The equations are solved numerically in Sec. III. The optical and dc conductivity are calculated in Secs. IV and V, respectively, making use of the Kubo formula. The results are discussed in Sec. VI.

\section{MODEL AND SELF-CONSISTENT SOLUTION}

We consider the following Holstein-Coulomb Hamiltonian:

$$
\begin{aligned}
H= & -\frac{t}{\sqrt{z}} \sum_{\langle i j\rangle}\left(c_{i}^{\dagger} c_{j}+\text { H.c. }\right)+\frac{1}{2} k X_{i}^{2}+g \sum_{i}\left(c_{i}^{\dagger} c_{i}-n\right) X_{i} \\
& +\frac{V}{2 z} \sum_{\langle i j\rangle}\left(n_{i}-n\right)\left(n_{j}-n\right) .
\end{aligned}
$$

where the operator $c_{i}^{\dagger}\left(c_{i}\right)$ creates (destroys) a spinless electron at site $i, n$ is the average electron density per site, and $X_{i}$ are the displacements of local oscillator modes, which are coupled to the local electron density via the parameter $g$. In addition to this electron-phonon interaction à la Holstein, the electrons interact mutually via a nearest neighbor repulsion term $V$ of Coulomb origin. The scaling of the repulsive term is chosen in such a way that the energy cost for a deviation from perfect charge ordering (i.e., flipping an occupied and an empty site) equals $V$. The scaling of the kinetic term $t / \sqrt{z}$ yields a finite free-electron bandwidth in the limit of infinite connectivity (number of nearest neighbors $z \rightarrow \infty$ ). We shall consider for simplicity a semi-elliptical density of states (DOS) of half-width $D=2 t$, corresponding to a Bethe lattice of infinite connectivity. Nevertheless, the present calculation scheme can be easily generalized to other model DOS to include more realistic band structures, as obtained, for example, from $a b$ initio calculations of specific systems. We shall specialize to the commensurate concentration $n=1 / 2$, which is equivalent to a quarter-filled band in the case of electrons with spin. In the present spinless case, the chemical potential is fixed at $\mu=0$ from particle-hole symmetry.

An order parameter for the $\mathrm{CO}$ transition can be defined as the charge disproportionation,

$$
\Delta n=n_{A}-n_{B},
$$

which varies between 0 and 1 , the average density being given by $n=\left(n_{A}+n_{B}\right) / 2=1 / 2$ (here, $A$ and $B$ label, respectively, the charge-rich and charge-poor sublattices). The formalism needed to treat the electron-phonon interaction was set up in Ref. 23 by mapping the original lattice problem 


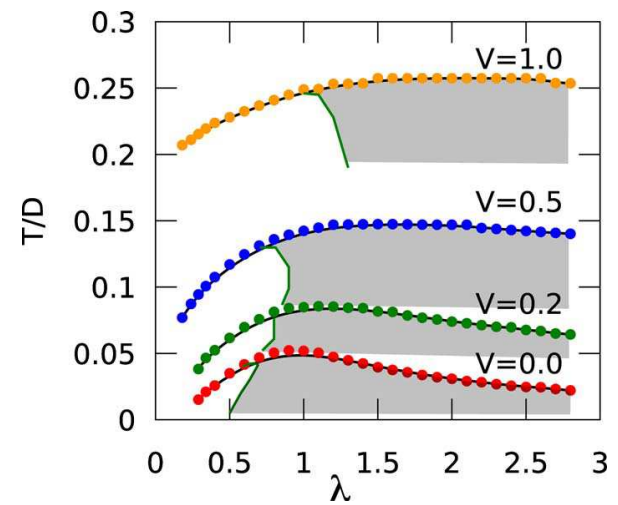

FIG. 1. (Color online) Critical temperature delimiting the CO phase as a function of $\lambda=g^{2} / 2 k D$ for different values of $V / D$. The shaded areas below $T_{c}$ mark polaronic regions where defects are present. The existence of defects is tracked by looking at metastable minima of the adiabatic potentials $V_{A, B}=-T \log \left(P_{A, B}\right)$.

onto a pair of coupled impurity models. In the limit of infinite connectivity, any additional nonlocal interaction term self-averages to its mean field (Hartree) value. Therefore, the extra Coulomb term can be straightforwardly included via a site dependent chemical potential shift in the Weiss fields, i.e., the local fields that take into account the effects of the electron itinerancy. On the Bethe lattice, the new Weiss fields read

$$
\begin{aligned}
& \left(G_{0}^{-1}\right)^{A}=i \omega_{n}+\frac{V}{2} \Delta n-\frac{D^{2}}{4} G^{B B}, \\
& \left(G_{0}^{-1}\right)^{B}=i \omega_{n}-\frac{V}{2} \Delta n-\frac{D^{2}}{4} G^{A A} .
\end{aligned}
$$

It can be observed by direct inspection of Eqs. (3) and (4) that the Coulomb interaction does not affect the properties of the system in the normal phase (where $\Delta n=0$ ), which is a drawback of the present approximation.

The above system of equations is closed self-consistently by expressing the site-diagonal propagators $G^{A A}$ and $G^{B B}$ in terms of the self-energy arising from the local interaction with the static phonon field,

$$
G^{A A}=\int d X \frac{P_{A}(X)}{\left(G_{0}^{-1}\right)^{A}-g X},
$$

and similarly for $G^{B B}$. Here, $P_{A}(X)$ and $P_{B}(X)$ are the probability distribution functions (PDFs) for the phonon displacements on the $A$ and $B$ sublattices, which can be obtained by tracing out the electronic degrees of freedom s $^{23}$

$$
P_{A}(X) \propto e^{-\beta k X^{2} / 2} \prod_{n}\left[\left(G_{0}^{-1}\right)^{A}-g X\right] e^{i \omega_{n} 0^{+}} .
$$

The numerical solution is achieved by successive iterations of the above equations, starting from a given ansatz for the sublattice propagators $G^{A A}$ and $G^{B B}$. In the following, we shall take the half-bandwidth $D$ as the unit of energy.

We now briefly analyze the phase diagram obtained from the DMFT solution of the model [Eq. (1)]. Figure 1 shows the critical temperature for the charge ordering transition as a function of the electron-phonon coupling parameter $\lambda$ $=g^{2} / 2 k D$, defined as the polaron energy $E_{P}=g^{2} / 2 k$ in units of the half-bandwidth. At $V=0$, we recover the bell-shaped result obtained in Ref. 23. Such bell shape originates from the different mechanisms that drive the charge ordering in the two limiting regimes of small and large $\lambda$. At weak coupling, the critical temperature monotonically increases with the electron-phonon coupling, i.e., the same qualitative trend predicted by the usual BCS theory. ${ }^{27}$ In this regime, the charge disproportionation and the long-range order take place simultaneously at $T_{c}$. At strong coupling instead, due to polaron formation, a local charge segregation corresponding to the localization of the electrons on randomly distributed sites occurs at a temperature $T \approx E_{P}$, much larger than ordering temperature $T_{c}$ itself. The actual critical temperature $T_{c}$ marks the onset of spatial ordering of such randomly distributed polarons. It is proportional to the "charge superexchange" $J=D /(4 \lambda)$ and therefore decreases as the electronphonon coupling increases. The asymptotic strong-coupling expression for the critical temperature is $T_{c}=D /(16 \lambda)$. From the above discussion, we see that the maximum of $T_{c}$, obtained at $\lambda \approx 1$ for $V=0$, signals the crossover between the weak coupling and the polaronic behavior.

The ordered phase below $T_{c}$ is also qualitatively different depending on the value of the electron-phonon coupling. In particular, in the strong-coupling regime, the preexisting polarons progressively order upon lowering the temperature and only attain a perfect alternate order at $T=0$. At any finite temperature, a certain number of defects exist within the $\mathrm{CO}$ phase in the form of charges localized on the "wrong" sublattice. The existence of such thermally induced defects is intimately related to the existence of local lattice distortions in the normal phase, i.e., polaron formation. ${ }^{23}$ We can therefore identify the existence of a polaronic $\mathrm{CO}$ phase, as opposed to the weak-coupling charge density wave, as the phase in which these defects are present.

The defect phase can be quantitatively characterized by analyzing the sublattice PDFs, which acquire a bimodal structure for sufficiently large $\lambda$. More efficiently, one can look for the existence of two nondegenerate minima in the sublattice adiabatic potentials defined as $V_{A, B}=-T \log P_{A, B}$. The result of this procedure is illustrated by the shaded areas in the phase diagram of Fig. 1. We see that the defect phase emerges right at the polaron crossover, i.e., in the region below the maximum of $T_{c}$ vs $\lambda$.

Including a direct intersite repulsion clearly favors both types of charge ordering, as it increases $T_{c}$ for all values of $\lambda$ (compare the different curves in Fig. 1). Still, the evolution of the phase diagram with $V$ suggests a nontrivial interplay between the direct Coulomb repulsion and the polaronic physics, as a finite $V$ shifts the polaron crossover (as well as the boundary of the defect phase) toward larger values of $\lambda$. It can be argued that, by treating the intersite Coulomb interaction beyond mean field, charge fluctuations of the same nature of the lattice defects evidenced above could start playing a role. ${ }^{28}$ For this reason, in the following, we shall restrict to the regime of small to moderate $V$, where the dominant physics is set by the electron-phonon interactions, therefore suppressing the fluctuations of the Coulomb interaction term. 


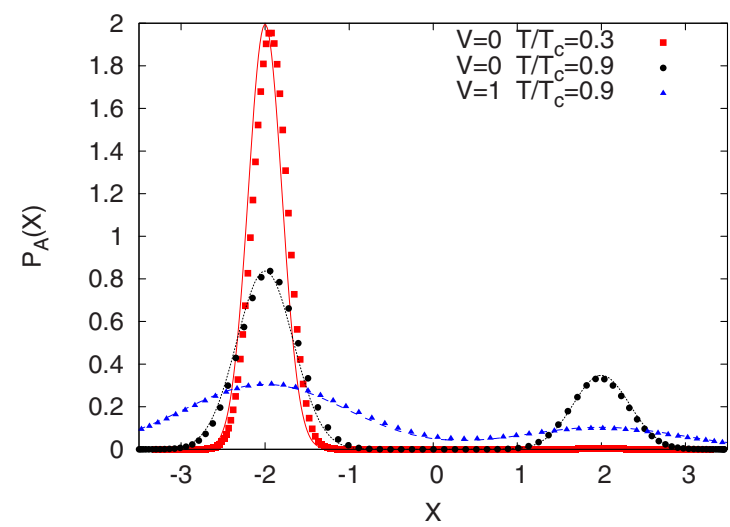

FIG. 2. (Color online) Sublattice phonon PDF at $\lambda=2$. The different curves are for $V=0\left(T / T_{c}=0.3\right.$ and 0.9$)$ and $V=1\left(T / T_{c}\right.$ $=0.9$ ). The points correspond to the DMFT self-consistent solution and the lines represent the strong-coupling theory [Eq. (B5)]. The locus and shape of the peaks do not depend explicitly on $V$.

The sublattice PDF $P_{A}(X)$ evaluated at $\lambda=2$, well inside the polaronic regime, is shown in Fig. 2. Defects in the $A$ sublattice give rise to a minority peak of the opposite "polarization" with respect to the preferred one. The area of the minority peak can be used to define the number of defects $\left(n_{d}\right)$ in the CO phase. In the strong-coupling regime, it tends to $1 / 2$ at $T=T_{c}$, where the two peaks become equivalent (polarons are randomly distributed over the two sublattices), while it vanishes exponentially at $T=0$, when perfect charge ordering is achieved. This occurs because as the temperature is reduced, the population of the metastable minimum of the potentials $V_{A, B}$ is progressively depleted, leading to an exponential reduction in $n_{d}$. It should be noted that the possibility of strictly $n_{d}=0$ is related to our classical treatment of the lattice degrees of freedom, which breaks down at temperatures much smaller than the characteristic phonon energies. Properly including phonon quantum fluctuations ${ }^{29}$ would lead to the appearance of charge defects even in the zero temperature limit.

In the limit $\lambda \rightarrow \infty$, the number of defects is related to the order parameter through

$$
\Delta n=1-2 n_{d} .
$$

At finite $\lambda$ in the polaronic phase $(\lambda \gtrsim 1)$, Eq. (7) above has to be replaced by $\Delta n=\left(1-2 n_{d}\right)\left[1-1 /\left(8 \lambda^{2}\right)+\cdots\right]$, which properly accounts for the fact that the charge disproportionation $\Delta n$ does not strictly tend to 1 even when the polarons are perfectly ordered $\left(n_{d}=0\right)$. This is because at finite $\lambda$, the electronic wave function is not fully localized and acquires a finite extension on the neighboring sites, which necessarily pertain to the minority sublattice.

The evolution of the charge disproportionation with $T$ in different regions of the phase diagram is reported in Fig. 3. In the polaronic regime, $\Delta n$ is a universal function of $T / T_{c}$, independent of $V$, as shown in Fig. 3(b). This means, in particular, that the number of defects $n_{d}$ is a function of $T / T_{c}$ alone, and $V$ only enters implicitly through the determination of $T_{c}$. Interestingly, the gap ratio $\Delta_{T=0} / T_{c}$ can become very large in the polaronic regime due to the pre-existing

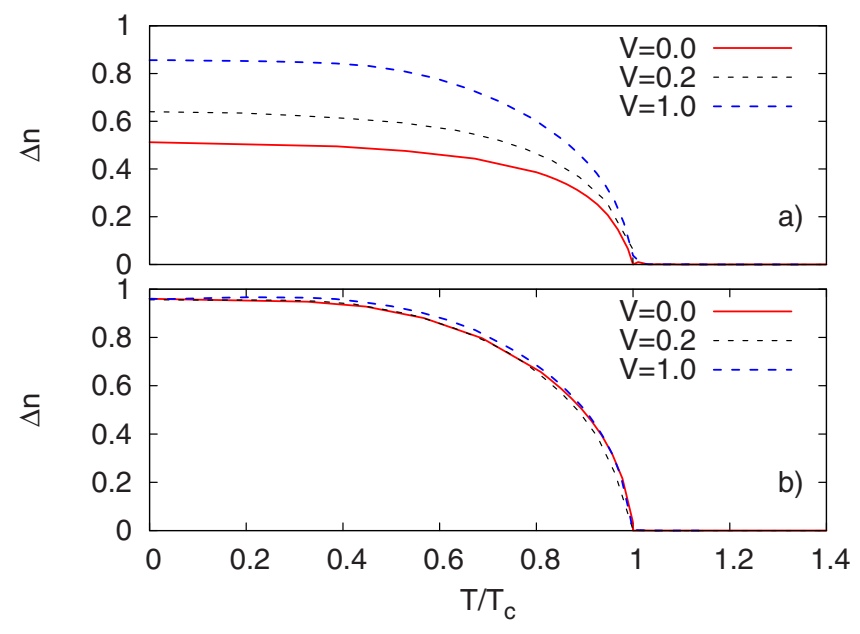

FIG. 3. (Color online) Evolution of the charge disproportionation for (a) $\lambda=0.4$ and for (b) $\lambda=2.0$ at different values of $V$.

pseudogap $\Delta \propto E_{P} \gg T_{c}{ }^{23}$ In the opposite weak electronphonon coupling limit, we recover a BCS-like behavior for $\Delta n$. In that case, the gap is given by $\Delta=(\lambda D+V / 2) \Delta n$. Its value at $T=0$ satisfies the mean field ratio $\Delta_{T=0} / T_{c}=3.54$. It is worth noting that this limit is only reached asymptotically as $\lambda \rightarrow 0$. For small but finite $\lambda, T_{c}$ is dramatically reduced by thermally induced lattice fluctuations, ${ }^{23,30}$ while $\Delta_{T=0}$ is not. Therefore, the gap $/ T_{c}$ ratio rapidly increases with $\lambda$ even well outside the polaronic region.

\section{OPTICAL CONDUCTIVITY}

\section{A. Dynamical mean field theory formulation}

We now derive the Kubo formula for the optical conductivity in the $\mathrm{CO}$ phase. To this aim, we develop a general formalism which makes explicit use of translational invariance as in the hypercubic lattice, following the lines of Ref. 31. In this framework, it is possible to write the expressions for the electron propagators in $k$ space. This is achieved through a canonical transformation that defines new electron creation operators $c_{\mathbf{k}}^{A, B}=\left(c_{\mathbf{k}} \pm c_{\mathbf{k}+\mathbf{Q}}\right) / \sqrt{2}$, where $\mathbf{Q}$ $=(\pi, \pi, \ldots)$ is the instability wave vector in any dimensions and $\mathbf{k}$ spans the reduced Brillouin zone (RBZ). With the above transformation, the tight binding term becomes $\sum_{k}^{\mathrm{RBZ}} \epsilon_{\mathbf{k}}\left(c_{\mathbf{k}}^{\dagger A} c_{\mathbf{k}}^{B}+c_{\mathbf{k}}^{\dagger B} c_{\mathbf{k}}^{A}\right)$, where $\epsilon_{\mathbf{k}}$ is the original noninteracting band dispersion. The fully interacting fermion propagators on the bipartite lattice can be defined as the matrix elements,

$$
G_{\mathbf{k}}^{\alpha \beta}=-i\left\langle T c_{\mathbf{k}}^{\alpha}(t) c_{\mathbf{k}}^{\beta}(0)\right\rangle \quad(\alpha, \beta=A, B),
$$

of the $2 \times 2$ matrix $\hat{G}_{\mathbf{k}}$. The corresponding spectral functions are given by $\hat{\rho}(\mathbf{k}, \omega)=-\operatorname{Im} \hat{G}_{\mathbf{k}}(\omega) / \pi$. Once the local selfenergies on the two sublattices are known from the solution outlined in Sec. II, the Green's functions of Eq. (8) are obtained by inverting the matrix,

$$
\hat{G}_{\mathbf{k}}^{-1}=\left(\begin{array}{cc}
z^{A} & -\epsilon_{\mathbf{k}} \\
-\epsilon_{\mathbf{k}} & z^{B}
\end{array}\right),
$$

with $z^{A}=\omega+i \delta+\mu-\Sigma_{A}(\omega)$, and similarly for $z^{B}$. 
In the tight binding model of Eq. (1), the current operator along a given (say, $x$ ) direction reads

$$
J_{x}=-i \frac{t}{\sqrt{z}} \sum_{i, \hat{\delta}} \delta_{x} c_{i+\hat{\delta}}^{\dagger} c_{i}
$$

where the sum extends over all sites $i$ of the lattice and their $z$ nearest neighbors, identified by the vectors $\hat{\delta}$. Transforming to the sublattice operators, the current operator can be expressed as

$$
J_{x}=-i \sum_{\mathbf{k} \in \mathrm{RBZ}} v_{\mathbf{k}}\left(c_{\mathbf{k}}^{\dagger A} c_{\mathbf{k}}^{B}+c_{\mathbf{k}}^{\dagger B} c_{\mathbf{k}}^{A}\right)
$$

In a hypercubic lattice, only the two neighbors along the $x$ direction contribute to the above sum, and the corresponding current vertex is $v_{\mathbf{k}}=2 \frac{t}{\sqrt{z}} \sin k_{x}$.

In the context of DMFT, due to the vanishing of vertex corrections, ${ }^{32-34}$ the current-current correlation function can be expressed exactly in terms of single particle Green's functions. This simplification still holds in the broken-symmetry phase because the $\mathbf{k} \rightarrow-\mathbf{k}$ symmetry is preserved even in the reduced Brillouin zone. Reminding the definitions in Eq. (8), we can write the current-current correlation function as

$$
\left\langle T J_{x}(t) J_{x}(0)\right\rangle=\sum_{\mathbf{k} \in \mathrm{RBZ}} v_{\mathbf{k}}^{2} \operatorname{Tr}\left[\tau_{x} \hat{G}_{\mathbf{k}}(t) \tau_{x} \hat{G}_{\mathbf{k}}(-t)\right],
$$

where we have made use of the Pauli matrix $\tau_{x}$ to obtain a compact expression. The trace is performed in the sublattice indices $A, B$.

The optical conductivity follows from the Kubo formula, upon transforming the current-current correlation function to the frequency domain,

$$
\begin{aligned}
\sigma(\omega)= & \sigma_{0} \int_{\mathrm{RBZ}} d \epsilon N(\epsilon) \phi(\epsilon) \int_{-\infty}^{\infty} d \nu \operatorname{Tr}\left[\tau_{x} \hat{\rho}(\epsilon, \omega+\nu) \tau_{x} \hat{\rho}(\epsilon, \nu)\right] \\
& \times \frac{f(\nu)-f(\nu+\omega)}{\omega} .
\end{aligned}
$$

In the above equation, the constant $\sigma_{0}=\pi e^{2} a^{2} / \hbar v$ carries the dimensions of conductivity, with $a$ as the lattice spacing, $v$ as the volume of the unit cell, $f(\nu)=\left[1+e^{\beta(\nu-\mu)}\right]^{-1}$ as the Fermi function, and $\hat{\rho}$ as the spectral functions associated with sublattice propagators $\hat{G}$. Taking advantage of the local nature $(k$ independence) of the self-energy, the sum over momenta in Eq. (12) has been replaced by an integration over energies, weighted by the DOS $N(\epsilon)$ and the current vertex $\phi(\epsilon)$ of the noninteracting lattice. Use has also been made of the fact that $v_{\mathbf{k}}^{2}$ is invariant under the transformation $\mathbf{k} \rightarrow \mathbf{k}+\mathbf{Q}$. The integration over the band dispersion $\epsilon$ in the current-current correlation function can be performed analytically, leading to

$$
\sigma(\omega)=\sigma_{0} \int_{-\infty}^{\infty} d \nu B(\nu+\omega, \nu) \frac{f(\nu+\omega)-f(\nu)}{\omega} .
$$

The function $B$ is defined as

$$
B(\nu+\omega, \nu)=-\frac{1}{4 \pi} \operatorname{Re}\left\{\chi\left(z_{1}^{A}, z_{1}^{B} ; z_{2}^{A}, z_{2}^{B}\right)-\chi\left(z_{1}^{A}, z_{1}^{B} ; z_{2}^{A *}, z_{2}^{B *}\right)\right\}
$$

where

$$
\begin{aligned}
\chi\left(z_{1}^{A}, z_{1}^{B} ; z_{2}^{A}, z_{2}^{B}\right)= & \frac{2}{\xi_{1}^{2}-\xi_{2}^{2}}\left\{\frac{\mathcal{K}\left(\xi_{2}\right)}{\xi_{2}}\left[z_{1}^{B} z_{2}^{A}+z_{1}^{A} z_{2}^{B}+2 \xi_{2}^{2}\right]\right. \\
& \left.-\frac{\mathcal{K}\left(\xi_{1}\right)}{\xi_{1}}\left[z_{1}^{B} z_{2}^{A}+z_{1}^{A} z_{2}^{B}+2 \xi_{1}^{2}\right]\right\},
\end{aligned}
$$

and

$$
\begin{gathered}
z_{1}^{\alpha}=\omega+\nu+i \delta-\Sigma_{\alpha}(\omega+\nu), \\
z_{2}^{\alpha}=\nu+i \delta-\Sigma_{\alpha}(\nu), \\
\xi_{1}=\sqrt{z_{1}^{A} z_{1}^{B}} \\
\xi_{2}=\sqrt{z_{2}^{A} z_{2}^{B}}
\end{gathered}
$$

with $\alpha=A, B$ the sublattice index. The function $\mathcal{K}$ is the Hilbert transform of the product $N(\epsilon) \phi(\epsilon)$, which can be evaluated analytically in the case of a semi-elliptical DOS. For this, we take the following form of the current vertex $\phi(\epsilon)$ $=\left(D^{2}-\epsilon^{2}\right) / 3$. This is chosen in such a way as to fulfill the $f$-sum rule, ${ }^{35-37}$ which relates the total optical spectral weight,

$$
W=\int_{0}^{\infty} d \omega \sigma(\omega)
$$

to the kinetic energy $K$ of the interacting system. The demonstration of the $f$-sum rule in the broken-symmetry phase is explicitly carried out in Appendix C.

In Secs. III B and III E, we shall analyze separately the results in the weak electron-phonon coupling regime $\lambda \lesssim 1$, in the extreme strong-coupling limit $\lambda \gg 1$, and in the most interesting polaronic regime $\lambda \gtrsim 1$.

\section{B. Weak coupling regime: $\lambda \lesssim 1$}

In Fig. 4, we report the results for the optical conductivity at $\lambda=0.4$ for different values of the intersite Coulomb interaction $V$. At such a moderate value of the electron-lattice coupling, although the overall behavior qualitatively agrees with what is expected in the conventional mean field scenario (see Appendix A, and the dashed lines in Fig. 4), the spectral features are appreciably smoothened by the presence of lattice fluctuations. A measure of such broadening is provided by the variance $s=\sqrt{2 E_{P} T}$ of the energy fluctuations of the phonon field, ${ }^{38,39}$ which is of the order of $0.1 D$ in the example of Fig. 4. This is a sizable fraction of the electronic bandwidth, which is, however, sufficiently small that the overall band picture remains qualitatively valid at temperatures comparable or below $T_{c}$, which are the ones of interest here.

Due to the classical approximation for the phonons, the Drude peak in the normal phase is replaced by an incoherent 


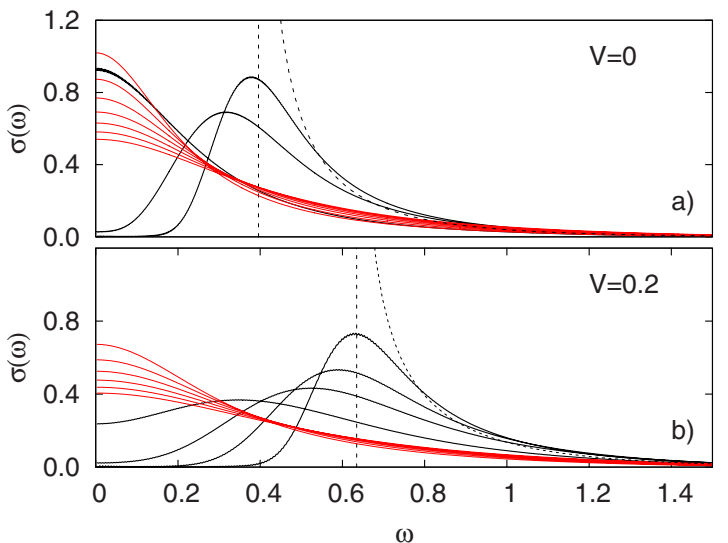

FIG. 4. (Color online) Optical conductivity at (a) $\lambda=0.4, V=0$ and (b) $V=0.2 D$. The curves in panel (a) are at equally spaced temperatures in the range $T / T_{c}=0.4-2.9\left(T_{c}=0.026 D\right)$ and in panel (b) $T / T_{c}=0.2-1.5\left(T_{c}=0.052 D\right)$. The thin (red) curves correspond to the disordered phase at $T>T_{c}$. The dashed line is the mean field result $[\mathrm{Eq}$. (A2)] at the lowest temperature.

Gaussian peak around $\omega=0,{ }^{40}$ whose width is proportional to $s$. Below the critical temperature, a finite charge disproportionation develops (cf. Fig. 3), which is reflected in the optical spectra through a progressive gap opening. Correspondingly, the low-frequency spectral weight is transferred to frequencies above the optical gap located at $\Delta_{\mathrm{opt}} \simeq(2 \lambda D$ $+V) \Delta n$. The sharp square-root divergence at $\omega=\Delta_{\text {opt }}$ expected from the standard mean field treatment [dashed line, corresponding to Eq. (A2)] is also smoothened due to the presence of thermal lattice fluctuations. These are also responsible for the subgap absorption tail observed in Fig. 4, which is absent in the mean field result. As shown in Ref. 26, a similar broadening can also arise at $T=0$ due to quantum lattice fluctuations.

The effect of a finite $V$ is to strengthen the $\mathrm{CO}$ phase, without modifying the broadening of the spectral features. The optical gap increases both due to the increased value of the charge disproportionation $\Delta n$ (see Fig. 3) and to the explicit contribution $V \Delta n$, which represents the extra electrostatic cost to move one particle from one sublattice to the other.

\section{Strong coupling limit: $\lambda \gg 1$}

The results for the optical absorption in the strongcoupling limit are shown in Fig. 5 for $\lambda=2$. The behavior is very different from the weak-coupling regime analyzed previously. This is seen already in the normal phase, where the Drude peak is replaced by a broad peak at finite frequency. This peak arises due to the formation of small polarons and reflects the optical transitions within the polaron internal structure. The peak position scales with the polaron energy roughly as $\omega \approx 2 E_{P}$, and its width is again proportional to the spread $s$ of the lattice fluctuations.

Because the low-frequency spectral weight is already strongly suppressed in the normal phase, no clear gap opening is visible at $T_{c}$. Indeed, in Fig. 5(a) $(\lambda=2$ and $V=0)$, there is no visible depletion of the optical conductivity at low fre-

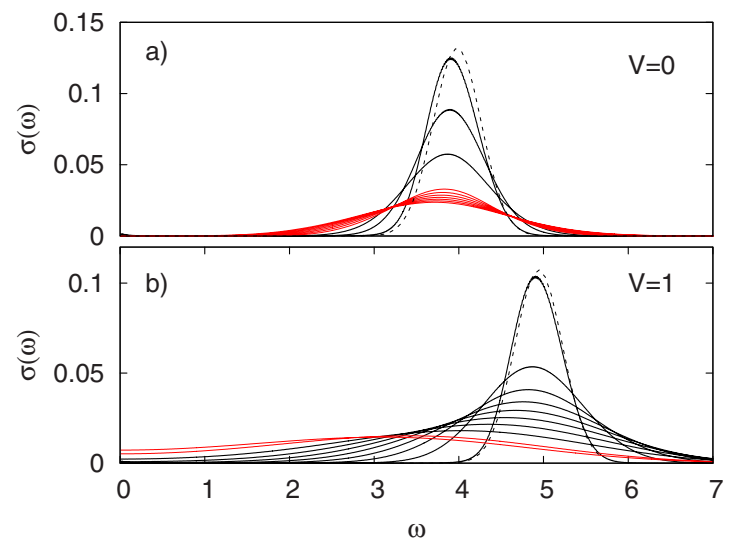

FIG. 5. (Color online) Optical conductivity at (a) $\lambda=2, V=0$ and (b) $V=1$. The curves in panel (a) are at equally spaced temperatures in the range $T / T_{c}=0.3-2.5\left(T_{c}=0.031 D\right)$ and in panel (b) $T / T_{c}$ $=0.04-1.1 \quad\left(T_{c}=0.26 D\right)$. The thin (red) curves correspond to the disordered phase at $T>T_{c}$. The dashed line is the strong-coupling result $[$ Eq. (B7)] at the lowest temperature.

quency (apart from the natural evolution of the peak width governed by $s$ ) and no clear shift of the position of the polaron peak as the temperature is reduced below $T_{c}$. Rather, the ordering transition leads to a sharp increase of the spectral weight associated with the polaronic peak. As we demonstrate hereafter, this increase is a direct consequence of the suppression of charge defects and constitutes a distinctive signature of the polaronic charge ordering.

The observed behavior can be understood by noting that in the normal phase, only half of the sites neighboring a given polaron are unoccupied and therefore available for an optical transition, as induced by the current operator Eq. (10). The number of available empty neighbors increases as charge defects are progressively removed below $T_{c}$ and so does the weight of the polaronic absorption peak until each polaron becomes exclusively surrounded by unoccupied sites at $T=0$. The above analysis can be carried out, in general, to show that the probability for polaronic optical transitions is proportional to $n_{A}\left(1-n_{B}\right)$ (for polarons in sublattice $A$ ) and $n_{B}\left(1-n_{A}\right)$ (for polarons in sublattice $B$ ). The sum scales as $\left(1+\Delta n^{2}\right)$, leading to an increase of up to a factor of 2 of the optical spectral weight in the $\mathrm{CO}$ phase.

A more detailed discussion of the spectral weight enhancement is provided in Appendix B, where we derive the analytical expressions for the optical conductivity valid at large $\lambda$. We report here the formula appropriate in the $\mathrm{CO}$ phase at $V=0$, which is simply expressed in terms of the normal state polaron absorption $\sigma_{\text {norm }}(\omega)$ as

$$
\sigma(\omega)=2\left[1-2 n_{d}\left(1-n_{d}\right)\right] \sigma_{\text {norm }}(\omega) .
$$

This equation directly relates the observed enhancement of the polaron peak with the suppression of the number of defects, in agreement with the qualitative argument given above, as can be easily shown using Eq. (7) above. Both this expression and its analog [Eq. (B8)] valid at $V \neq 0$ describe quite accurately the full DMFT results at $\lambda=2$, as illustrated in Fig. 5. 

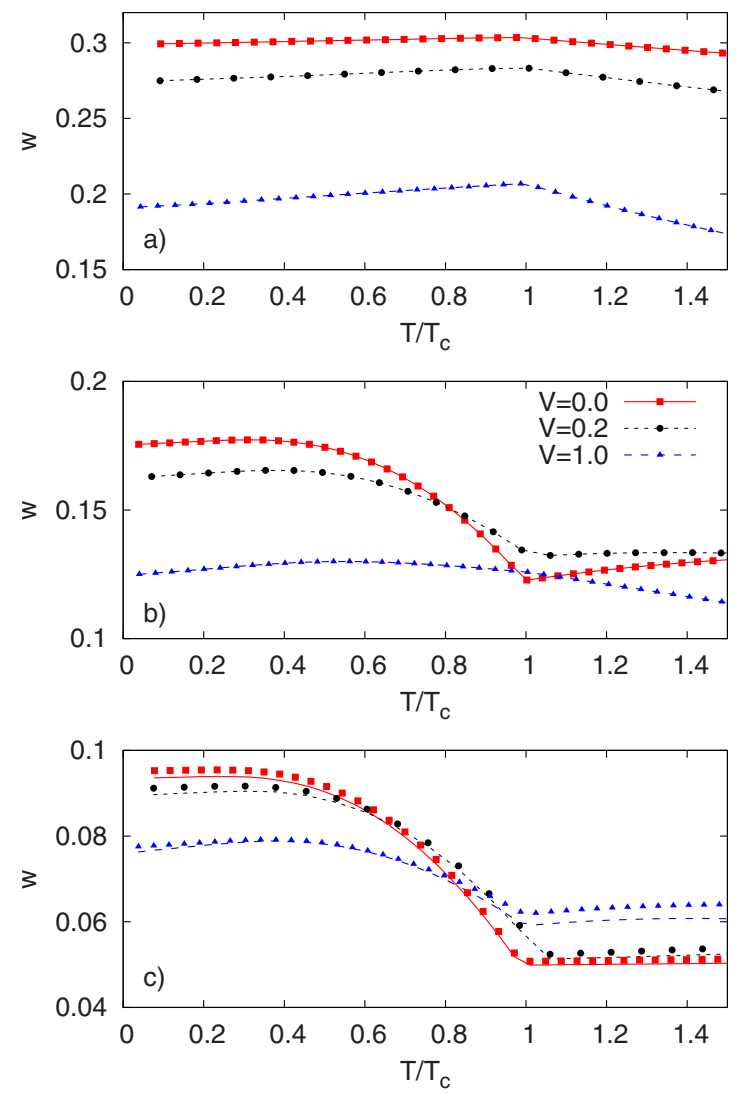

FIG. 6. (Color online) Total optical weight $W$ as defined in Eq. (21) for (a) $\lambda=0.4$, (b) $\lambda=1$, and (c) $\lambda=2$ at different values of $V$ (the legend is indicated in panel b). The lines in panels (a) and (b) are guides to the eye, while in panel (c), they correspond to the analytical strong-coupling approximation obtained by integrating Eqs. (B7) and (B8) down to a cutoff frequency equal to $T$.

\section{Spectral weight analysis}

The increase in spectral weight associated with the ordering of polarons is best visualized by analyzing the integrated optical conductivity $W$ defined in Eq. (21). The evolution of $W$ vs temperature is reported in Fig. 6 and exhibits a markedly different behavior at weak and strong electron-phonon couplings. In Fig. 6(c), corresponding to $\lambda=2$ and $V=0$, we see that upon lowering the temperature $W$ first decreases (in the normal phase) and then exhibits a sharp increase (in the ordered phase), saturating at $T \ll T_{c}$, to a value that is about twice the normal phase value. Therefore, at large $\lambda$, the critical temperature corresponds to a minimum of $W$. This remains true in all the polaronic regime, down to the polaron crossover at $\lambda \sim 1$. This is opposite to the usual behavior of charge density waves, where upon lowering the temperature the optical weight first increases and then decreases, reaching a maximum at the critical temperature. Such conventional behavior is recovered at small $\lambda$, as illustrated in Fig. 6(a) for $\lambda=0.4$.

These opposite behaviors can be understood in virtue of the optical sum rule demonstrated in Appendix C, which relates the total spectral weight to (minus) the total kinetic energy $K$ of the system. The latter is usually expressed as $K=\Sigma_{k} \epsilon_{k} n_{\epsilon_{k}}$, where $n_{\epsilon_{k}}$ is the momentum distribution function
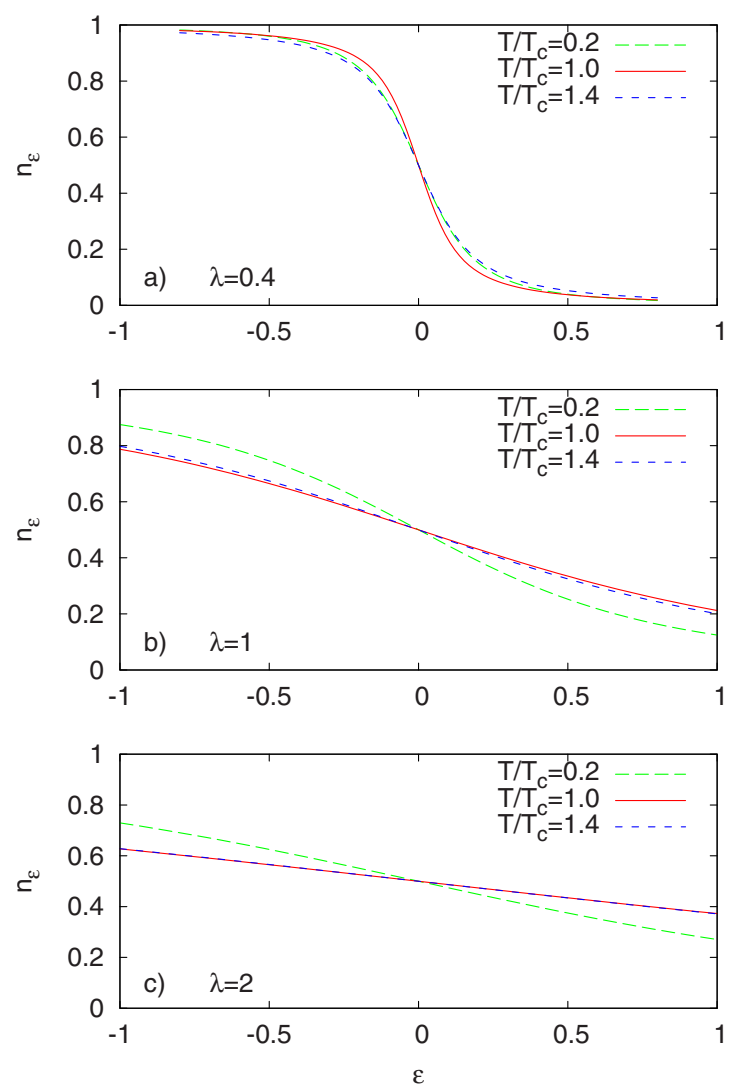

FIG. 7. (Color online) Momentum distribution function vs band energy $\epsilon=\epsilon_{k}$ at temperatures at, below, and above $T_{c}$. The coupling strengths are the same as in Fig. 6 , and we have set $V=0$.

of the interacting electrons. As shown in Appendix C, an equivalent expression holds true even in the brokensymmetry phase [see Eq. (C7)] if one properly introduces the occupation number for the generalized propagator $\bar{G}$ $=1 /\left[\sqrt{z_{A} z_{B}}-\epsilon_{k}\right]$. Plots of this quantity are shown in Fig. 7 .

As pointed out in Ref. 41, any broadening of the step feature in $n_{\epsilon_{k}}$ around the Fermi energy, by populating states with higher energy, leads to an increase in $K$ and therefore to a decrease of the spectral weight $W$. In the case of a conventional charge density wave, illustrated in Fig. 7(a), the distribution $n_{\epsilon_{k}}$ broadens both upon increasing the temperature above $T_{c}$ and upon opening a gap below $T_{c}$. As a result, the critical temperature identifies a minimum of $K$, i.e., a maximum of $W$.

The situation is different in the strong electron-phonon coupling regime [Figs. 7(b) and 7(c)], where the formation of small (local) polarons involves all the states in the Brillouin zone. Correspondingly, the distribution $n_{\epsilon_{k}}$ becomes extremely flat. In this case, increasing the temperature above $T_{c}$ progressively destroys the local correlations that build up the polaron, and the momentum distribution eventually recovers some structure at the Fermi level, leading to a decrease in $K$ (the effect is small because $T_{c}$ in this regime is much smaller than the energy scale $E_{P}$ that governs the polaron dissociation). The polaron ordering below $T_{c}$ also decreases $K$ because the emergence of two distinct sublattices effectively restores some momentum-space structure. In the polaronic 


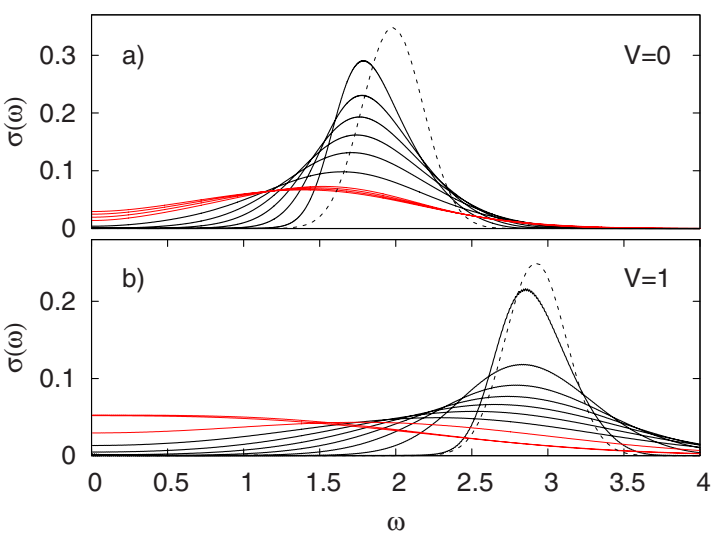

FIG. 8. (Color online) Optical conductivity at (a) $\lambda=1, V=0$ and (b) $V=1.0$. The curves in panel (a) are at equally spaced temperatures in the range $T / T_{c}=0.2-1.5\left(T_{c}=0.052 D\right)$ and in panel (b) $T / T_{c}=0.04-1.1\left(T_{c}=0.25 D\right)$. The curves corresponding to the disordered phase at $T>T_{c}$ are in red. The dashed line is the strongcoupling result $[\mathrm{Eq}$. (B7)] at the lowest temperature.

regime, the critical temperature therefore defines a maximum of $K$, i.e., a minimum of $W$.

\section{E. Polaronic regime: $\lambda \gtrsim 1$}

By following the evolution of the spectral weight from Figs. 6(a)-6(c), it is interesting to see that at intermediate values of the electron-lattice coupling (but still in the polaronic regime), the two competing trends evidenced above lead to a nonmonotonic temperature dependence of the $\mathrm{ki}$ netic energy (and of the spectral weight) within the $\mathrm{CO}$ phase. This can be seen, in particular, the curve labeled $V$ $=0$ at $\lambda=1$ in Fig. 6(b). Note that a nonmonotonic behavior of the kinetic energy analogous to the one described here was recently observed in the ordered phase of the FalicovKimball model at intermediate $U .{ }^{25}$

The spectral weight analysis presented above suggests that both features characteristic of the weak- and strongcoupling limits coexist in the polaronic regime at intermediate coupling strengths $\lambda \gtrsim 1$. We can check in Fig. 8(a) $(\lambda$ $=1$ and $V=0)$ that such coexistence also naturally manifests in the optical spectra. Here, as in the strong-coupling limit of Fig. 5(a), the polaronic nature of the carriers is testified by the presence of a broad finite-frequency peak already in the normal phase. In this case, however, where the polaron energy $E_{P}$ is comparable to the free electronic bandwidth $D$, the peak position lies well below the strong-coupling estimate $2 E_{P}$. Also, the shape and width of the peak deviate from the strong-coupling estimate, being both controlled by the ratio $s / D$ (the usual symmetric Gaussian shape is recovered as $s \gg D$, see Ref. 37). Most interesting, however, is the evolution of the optical absorption below $T_{c}$, which shares similarities with both behaviors shown in Figs. 4(a) and 5(a). Indeed, at intermediate values of the electron-phonon coupling, the optical absorption in the ordered phase exhibits both a marked enhancement and an appreciable transfer of spectral weight to higher frequencies. This composite behavior gives rise to an "isosbestic" region at frequencies below the polaron peak, where the optical absorption is almost independent of temperature.

It was observed by analyzing the phase diagram in Sec. II that the intersite repulsion $V$ effectively pushes the system toward the weak electron-lattice coupling regime. This conclusion is also supported by the behavior of the optical conductivity spectra. For example, we see from Fig. 1 that at $\lambda=1$, a repulsion $V=1$ is sufficient to move the system outside the polaronic phase. Accordingly, the polaron peak that was present at high temperature in Fig. 8(a) has disappeared in the $V=1$ spectrum of Fig. 8(b), and a more conventional gap opening is restored below $T_{c}$. If instead one starts from the strong-coupling value $\lambda=2$, an appreciable transfer spectral of spectral weight below the transition is recovered for $V=1$, as well as a nonmonotonic behavior of the total spectral weight $W$ [see Figs. 5(b) and 6(c)].

\section{IV. de CONDUCTIVITY}

In this section, we briefly describe the dc conductivity, which is obtained from the previously derived Kubo formula [Eq. (13)] by taking the limit $\omega \rightarrow 0$. The numerical results for $\sigma_{\mathrm{dc}}$ across the $\mathrm{CO}$ transition are shown in Fig. 9 for different values of $V$ at (a) $\lambda=0.4$, (b) $\lambda=1$, and (c) $\lambda=2$. These values are the same as analyzed in Sec. III E and correspond, respectively, to the weak, to the intermediate, and to the strong electron-lattice coupling regime. The CO transition can be clearly identified as a knee in all the conductivity curves, except for $\lambda=2$ and $V=0$ [in that case, $T_{c}$ falls outside the range of Fig. 9(c)]. In the weak-coupling regime [Fig. 9(a)], the opening of a gap changes the mobility from metalliclike to insulatinglike, i.e., the slope of $\sigma_{\mathrm{dc}}(T)$ changes sign. The changes in the transport properties at the $\mathrm{CO}$ transition become less marked in the polaronic regime. In this case, the mobility is already thermally activated in the normal phase, and the $\mathrm{CO}$ transition only causes an increase of the activation barrier, which is reflected in a change in slope in the Arrhenius plots of Figs. 9(b) and 9(c).

An expression for the dc conductivity valid in the strongcoupling limit is derived in Appendix B. In the normal phase, one recovers the usual formula for the polaronic activated behavior,

$$
\sigma_{\mathrm{dc}}^{\mathrm{norm}}(T)=\frac{\sigma_{0}}{32 \sqrt{2 \pi E_{P}} T^{3 / 2}} e^{-E_{P} / 2 T} .
$$

At temperatures below $T_{c}$, this generalizes to

$$
\sigma_{\mathrm{dc}}(T)=4 n_{d}\left(1-n_{d}\right) \sigma_{\mathrm{dc}}^{\mathrm{norm}}(T) .
$$

We see that the ordering transition reduces the conductivity in a way that is once again related to the suppression of defects, being proportional to the product of the occupations in the two sublattices, namely, $4 n_{d}\left(1-n_{d}\right) \simeq 1-\Delta n^{2}$.

It should be stressed that standard approaches that incorporate Coulomb interactions into the polaronic conductivity would predict an additive contribution $V \Delta n / 2$ to the activation barrier at the $\mathrm{CO}$ transition, ${ }^{42}$ which naturally arises from the extra energy cost that a polaron in the majority sublattice has to overcome to hop to a neighboring unoccu- 

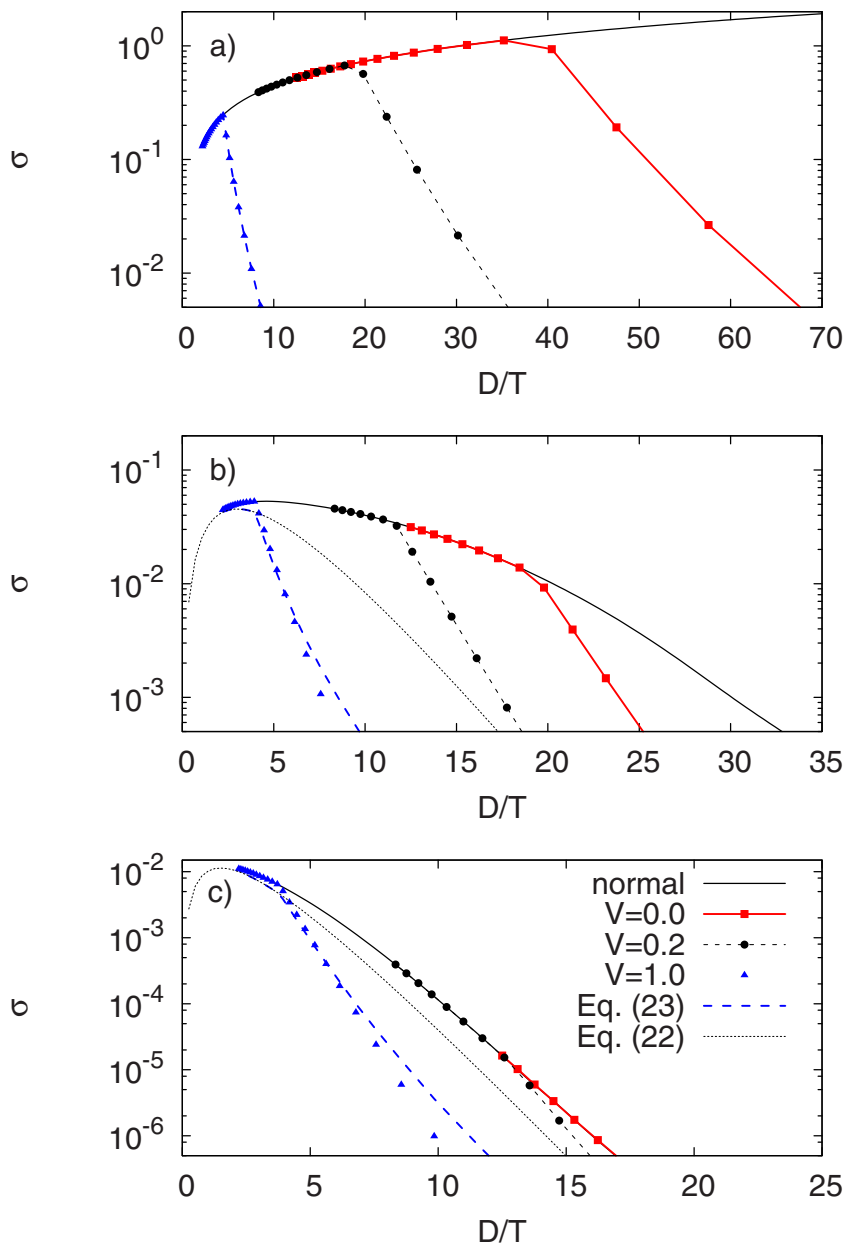

FIG. 9. (Color online) dc conductivity for (a) $\lambda=0.4$, (b) $\lambda=1$, and (c) $\lambda=2$ for different values of $V$. The lines are guides to the eye, except for $V=1$ in panels (b) and (c), where the line is the strong-coupling formula [Eq. (24)]. The full black line is the DMFT result obtained in the normal phase and the thin dashed line is the standard polaronic expression [Eq. (23)].

pied site. While such contribution is indeed recovered in our treatment [see Eq. (B13)], we see here that the dominant conduction mechanism at finite temperatures is related to the presence of charge defects. Being already thermally excited onto the minority sublattice, such defects do not pay an extra cost in Coulomb energy when hopping from site to site, and their motion is therefore more advantageous than ordinary hopping across the gap (remind that at large $\lambda$ the number of defects $n_{d}$ does not depend explicitly on $V$, as was shown in Fig. 3).

Equation (24) is compared with the full DMFT results for $\lambda=1, V=1$ and $\lambda=2, V=1$ in Figs. 9(b) and 9(c), respectively. It should be stressed that the strong-coupling analysis underlying Eqs. (23) and (24) above is strictly valid only at $\lambda \rightarrow \infty$, as it neglects corrections proportional to $D$ in the activation barrier. As a result, the above formulas widely underestimate the electrical conductivity, ${ }^{43}$ and the agreement with the DMFT data at finite $\lambda$ and $V=0$ is not as good as the one obtained for $\sigma(\omega)$ in Sec. III E. This is particularly evident when comparing Eq. (23) to the $\lambda=1$ data of Fig. 9(b), where large deviations arise especially at low tempera- tures $T \ll E_{P}$. Increasing $V$ shifts the ordering transition to higher temperatures, apparently improving the accuracy of the approximation.

\section{DISCUSSION AND CONCLUSIONS}

We have calculated the optical and electrical transport properties in a model describing charge ordering in systems with strong electron-lattice interactions. The DMFT treatment used here properly accounts for fluctuations of the lattice polarization. This allows us to understand the role played by charge defects in the electrodynamic response of the polaronic ordered phase. The effects of a moderate intersite Coulomb repulsion term, which is often present in interacting narrow-band systems, have also been incorporated.

In the weak electron-phonon coupling regime, we have seen in Sec. II that lattice fluctuations play an important role as soon as $\lambda>0$, as they strongly reduce the critical temperature compared to the mean field BCS-like expectation. ${ }^{23,30}$ Our results show that these fluctuations also manifest in the spectral properties, as they provide a disordered effective medium that scatters the electron motion, leading to an appreciable broadening of the sharp spectral features predicted by mean field theory.

The effects of lattice fluctuations are, however, much more dramatic in the strong-coupling regime, where the charge ordering transition occurs in a polaronic phase with a pre-existing charge localization. In that case, polaron formation moves most of the spectral weight to a finite frequency absorption peak, whose position hardly changes at the ordering transition. Instead, the distinctive signature of charge ordering in the strong-coupling limit is a marked enhancement of the polaronic absorption peak, which is directly related to the suppression of charge defects, i.e., charges promoted to the "wrong" sublattice by the thermal fluctuations. In the polaronic ordered phase, such defects also govern the transport properties, as their motion dominates over the usual hopping mechanism across the disproportionation gap. The competing scenarios described above appear to coexist at intermediate electron-lattice interactions $(\lambda \gtrsim 1)$, i.e., precisely in the region of the phase diagram where the critical temperature is maximum. In this region, polaronic charge ordering reveals in the optical spectra through both a transfer of spectral weight to high frequencies and a global spectral weight enhancement as the temperature is lowered below $T_{c}$.

It should be noted that a model very similar to the one studied here was developed in a series of papers of Ihle and Lorenz $\mathrm{z}^{42,44,45}$ and was applied to the long-standing problem of the Verwey transition in $\mathrm{Fe}_{3} \mathrm{O}_{4}$-a prototypical example of polaron ordering [see also the review paper (Ref. 22)]. In these works, the model [Eq. (1)] was solved relying on the antiadiabatic approximation for the lattice degrees of freedom, where the phonon dynamics is assumed to be faster than the electrons. Our adiabatic treatment can therefore be considered as complementary to the one of Refs. 44 and 45. While the present approach clearly gives more insight into the physics of charge defects, our classical treatment of phonons has an important limitation that one should keep in mind when attempting a comparison to actual experiments. 
The results obtained here are expected to apply at temperatures and frequencies larger than the frequency scale of the phonons involved in the $\mathrm{CO}$ transition. Properly accounting for the quantum nature of the phonons would restore the possibility of a coherent transport regime at low temperatures ${ }^{43}$ and allow for the presence of charge defects even at zero temperature. ${ }^{29}$

\section{ACKNOWLEDGMENTS}

The authors acknowledge useful discussions with L. Benfatto, E. Cappelluti, M. Dressel, G. Sangiovanni, and A. Toschi. S.F. is grateful for the kind hospitality of the Instituto de Ciencia de Materiales de Madrid, CSIC, where part of this work was done.

\section{APPENDIX A: MEAN FIELD TREATMENT OF THE OPTICAL CONDUCTIVITY}

We evaluate here the optical absorption in the $\mathrm{CO}$ state at mean field level, which applies to the weak electron-lattice coupling limit. This treatment also describes accurately the numerical data at any finite $V$ at nonzero temperature when $\lambda=0$, due to the intrinsic mean field treatment of nonlocal interactions in DMFT. The mean field solution for the electron propagators is obtained by inverting the matrix,

$$
\hat{G}_{\mathbf{k}}^{-1}=\left(\begin{array}{cc}
\omega+\Delta & -\epsilon_{k} \\
-\epsilon_{k} & \omega-\Delta
\end{array}\right),
$$

where $\Delta$ is the CO gap, which is determined self-consistently. ${ }^{23}$ It is related to the charge disproportionation by $\Delta=(\lambda D+V / 2) \Delta n$.

The corresponding spectral functions are delta functions, which allow for a direct evaluation of Eq. (13) for a generic DOS. The finite-frequency part, corresponding to interband transitions (across the gap), reads

$$
\sigma(\omega)=\frac{\sigma_{0}}{2} \frac{N \phi\left(\frac{1}{2} \sqrt{\omega^{2}-(2 \Delta)^{2}}\right)}{\sqrt{\omega^{2}-(2 \Delta)^{2}}} \frac{(2 \Delta)^{2}}{\omega^{2}} \tanh \left(\frac{\omega}{4 T}\right),
$$

which has a square-root singularity at $\omega=2 \Delta$ (the optical gap) followed by a power-law decay at higher frequencies. At finite temperature, states are thermally excited across the gap, which enables the possibility of intraband absorption. In the absence of additional inelastic scattering mechanisms, this gives rise to a zero-frequency peak,

$$
\sigma_{D}(\omega)=\sigma_{0} \delta(\omega) \int_{\mathrm{RBZ}} d \epsilon N(\epsilon) \phi(\epsilon) \frac{2 \epsilon^{2}}{\epsilon^{2}+\Delta^{2}}\left[-f^{\prime}\left(\epsilon^{2}+\Delta^{2}\right)\right] .
$$

\section{APPENDIX B: STRONG COUPLING APPROXIMATION TO $\sigma(\omega)$}

We derive here analytical approximations to Eqs. (5), (14), and (21) that are valid in the strong-coupling limit $\lambda$ $\gg 1$.

\section{Normal state}

Let us first note that the usual strong-coupling formula of $\mathrm{Reik}^{46}$ for the optical conductivity is recovered in the normal phase if we take the atomic limit $(t=0)$ in the propagators appearing in Eq. (13). Indeed, by setting $G_{0}^{-1}=\omega+i \delta$, it can be shown that the lattice PDF is a sum of two Gaussians centered at $\pm \mathrm{g} / 2 \mathrm{k}$, each one carrying a weight $n_{A}=n_{B}=1 / 2$ (Ref. 23) (as was mentioned above, the Coulomb interaction term does not affect the properties of the normal phase at mean field level). This can be cast in the form

$$
P_{\text {norm }}(X)=g \frac{\cosh (\beta g X / 2)}{\sqrt{4 \pi E_{P} T}} e^{-(g X)^{2}+E_{P}^{2} / 4 E_{P} T},
$$

with the polaron energy defined as $E_{P}=g^{2} / 2 k=\lambda D$. The spectral function is obtained straightforwardly from Eq. (B1) through the relation $\rho_{\text {norm }}(\omega)=P_{\text {norm }}(\omega / g) / g$ [cf. Eq. (5)].

Since in this limit the electron Green's function is site diagonal and momentum independent, the $\epsilon$ integration can be factored out from Eq. (13), yielding a prefactor $\int d \epsilon N(\epsilon) \phi(\epsilon)=1 / 4$ on the Bethe lattice. The remaining frequency integral can be performed using the relation

$$
[f(\omega+\nu)-f(\nu)]=\frac{\sinh \left[\frac{\beta \omega}{2}\right]}{2 \cosh \left[\frac{\beta(\omega+\nu)}{2}\right] \cosh \left[\frac{\beta \nu}{2}\right]},
$$

which leads to the desired result

$$
\sigma(\omega)=\frac{\sigma_{0}}{\sqrt{2 \pi E_{P} T}} \frac{\sinh (\beta \omega / 2)}{16 \omega} e^{-\left(\omega^{2}+4 E_{P}^{2} / 8 E_{P} T\right)} .
$$

Focusing on the finite frequency part $\omega \gg T$, this can be further simplified to

$$
\sigma_{\text {norm }}(\omega)=\frac{\sigma_{0}}{32 \omega \sqrt{2 \pi E_{P} T}} e^{-\left(\omega-2 E_{P}\right)^{2} / 8 E_{P} T} .
$$

This formula represents a modified Gaussian absorption band having its maximum at $\omega=2 E_{P}$ and a width $\sqrt{2} s=2 \sqrt{E_{P} T}$, which reflects the thermal fluctuations of the phonon field that couples to the electron motion. Note that if the phonon quantum fluctuations are correctly taken into account, the width of the absorption band does not shrink indefinitely at low temperatures but rather saturates to a finite value $s^{2}$ $=2 E_{P} \omega_{0} \cdot{ }^{37}$ One can observe that the numerical factor $1 / 32$ in Eq. (B4) implicitly includes the density dependent factor $n(1-n)$ expected from theories of independent polarons.

\section{Charge ordered state}

In the charge ordered state $(\Delta n \neq 0)$, the two contributions to the lattice PDF are unbalanced by a factor which takes into account the different fillings $n_{A} \neq n_{B}$ on nonequivalent sublattices. As a result, Eq. (B1) becomes

$$
P_{A, B}(X)=P_{\text {norm }}(X)\left[1 \pm\left(1-2 n_{d}\right) \tanh (\beta g X / 2)\right],
$$

where the + and - signs correspond to the $A$ and $B$ sublattices, respectively. The lattice PDF is again the sum of two Gaussian peaks, centered at the minima $\pm X_{0}$ of the adiabatic potentials $V_{A, B}$, whose weights are now, respectively, $n_{d}$ and 
$1-n_{d}$. At $\lambda=2$, this approximation compares very well with the full DMFT result illustrated in Fig. 2. That figure also shows that for moderate $V \lesssim 1$, the shape of the Gaussian peaks in the PDF is unaffected by the electrostatic repulsion, which only enters implicitly through the self-consistent determination of $n_{d}$.

From Eq. (B5), we obtain the spectral function for the two sublattices,

$$
\begin{aligned}
& \rho_{A}(\omega)=\left(1-n_{d}\right) g_{+}\left(\omega+\frac{V \Delta n}{2}\right)+n_{d} g_{-}\left(\omega+\frac{V \Delta n}{2}\right), \\
& \rho_{B}(\omega)=n_{d} g_{+}\left(\omega-\frac{V \Delta n}{2}\right)+\left(1-n_{d}\right) g_{-}\left(\omega-\frac{V \Delta n}{2}\right),
\end{aligned}
$$

where

$$
g_{\alpha}(\omega)=\frac{1}{\sqrt{2 \pi s^{2}}} \exp \left(-\frac{\omega-\alpha E_{P}}{2 s^{2}}\right),
$$

and $\alpha= \pm 1$.

Taking the low temperature limit $\left(T \ll \sqrt{E_{P} T}, D\right)$ in Eq. (13) and using the spectral functions above, it is possible to obtain an expression for the finite frequency conductivity $(\omega \gg T)$. At $V=0$, it takes the simple form

$$
\sigma(\omega)=2\left[1-2 n_{d}\left(1-n_{d}\right)\right] \sigma_{\text {norm }}(\omega),
$$

which directly relates the observed enhancement of the optical absorption in the $\mathrm{CO}$ phase to the number of charge defects, in agreement with the general arguments presented in Sec. III C. In the presence of intersite repulsion, the above formula generalizes to

$$
\begin{aligned}
\sigma(\omega)= & \frac{\sigma_{0}}{16 \omega \sqrt{2 \pi E_{P} T}}\left[e^{-\left(\omega-2 E_{P}-V \Delta n\right)^{2} / 8 E_{P} T}\left(1-n_{d}\right)^{2}\right. \\
& \left.+e^{-\left(\omega-2 E_{P}+V \Delta n\right)^{2} / 8 E_{P} T}\left(n_{d}\right)^{2}\right],
\end{aligned}
$$

which clearly reduces to Eq. (B7) when $V=0$. Both expressions compare well with the numerical data of Fig. 5 at $\lambda$ $=2$. Moreover, the total optical spectral weight obtained by integrating Eq. (B8) down to a cutoff frequency $\omega=T$ correctly reproduces the nonmonotonic temperature dependence of the DMFT results shown in Fig. 6(c). Note that at extremely large $V$, this expression predicts a double-peak structure, which is not observed in the numerical data at moderate $V$.

The results presented in this appendix, derived under the assumption of a vanishing bandwidth, are strictly valid as long as $s \gg D .{ }^{37}$ From the asymptotic strong-coupling expression $T_{c}=D /(16 \lambda)+V / 4$, we can estimate

$$
\left.\frac{s}{D}\right|_{T_{c}} \simeq \frac{1}{2^{3 / 2}} \sqrt{1+4 \lambda \frac{V}{D}}
$$

We see that at $V=0$, the condition $s \gg D$ is never realized in the relevant region around $T_{c}$, and some finite bandwidth corrections to the polaronic line shapes can be expected. ${ }^{37}$ Examination of Figs. 5 and 8 shows that the accuracy of the strong-coupling formula [Eq. (B7)] improves when an explicit Coulomb term $V$ is included.

\section{3. de conductivity}

The dc conductivity is obtained by taking the limit $\omega$ $\rightarrow 0$ in Eq. (13). Some care must be taken in integrating the Gaussian spectral functions in the presence of the factor $1 /[4 T \cosh (\nu / 2 T)]$, originating from the Fermi functions. For $T \ll E_{P}$, one obtains

$$
\begin{aligned}
\sigma_{\mathrm{dc}}(T)= & \frac{\sigma_{0}}{16 \pi E_{P} T} e^{-\left(E_{P} / 2 T\right)-(V \Delta n)^{2} / 8 E_{P} T}\left[2 n_{d}\left(1-n_{d}\right) \sqrt{\frac{\pi E_{P}}{2 T}}\right. \\
& \left.+\left(1-n_{d}\right)^{2} e^{-(V \Delta n / 2 T)}+n_{d}^{2} e^{V \Delta n / 2 T}\right] .
\end{aligned}
$$

which reduces in the normal phase to the usual formula for the polaronic conductivity,

$$
\sigma_{\mathrm{dc}}^{\mathrm{norm}}(T)=\frac{\sigma_{0}}{32 \sqrt{2 \pi E_{P}} T^{3 / 2}} e^{-\left(E_{P} / 2 T\right)} .
$$

In the $\mathrm{CO}$ state at temperatures $T \lesssim T_{c}$, the leading contribution to Eq. (B10) is

$$
\sigma_{\mathrm{dc}}(T)=4 n_{d}\left(1-n_{d}\right) e^{-(V \Delta n)^{2} / 8 E_{P} T} \sigma_{\mathrm{dc}}^{\mathrm{norm}}(T),
$$

which shows that the dominant conduction mechanism in the polaronic ordered phase involves thermally activated defects. The exponential term is close to 1 at moderate values of $V$ such as the ones studied here and can be dropped. This expression breaks down as $T \ll T_{c}$, when the number of defects $n_{d}$ vanishes. In this case, a more conventional result is recovered

$$
\sigma_{\mathrm{dc}}(T)=\frac{\sigma_{0}}{16 \pi E_{P} T} e^{-\left(E_{P}+V \Delta n / 2\right)^{2} / 2 E_{P} T} .
$$

This formula predicts an additive contribution $V \Delta n / 2$ to the activation barrier, corresponding to the extra energy cost that a polaron has to overcome when hopping to its neighboring sites. $^{42}$

\section{APPENDIX C: F-SUM RULE IN THE BROKEN- SYMMETRY PHASE}

In this section, we demonstrate the $f$-sum rule for the optical conductivity evaluated through the DMFT formula [Eq. (13)], generalizing the demonstration of Ref. 47 to the broken-symmetry phase. We first rewrite the integral of the optical conductivity in the form

$$
\int_{0}^{\infty} \sigma(\omega)=\frac{\sigma_{0}}{2} \int_{R B Z} d \epsilon N(\epsilon) \phi(\epsilon) I(\epsilon),
$$

with

$$
\begin{aligned}
I(\epsilon)= & \frac{1}{\pi^{2}} \int_{-\infty}^{\infty} d \nu \int_{-\infty}^{\infty} d \nu^{\prime} \frac{f(\nu)-f\left(\nu^{\prime}\right)}{\nu^{\prime}-\nu} \\
& \times \operatorname{Tr}\left[\sigma_{x} \operatorname{Im} \hat{G}_{\epsilon}\left(\nu^{\prime}\right) \sigma_{x} \operatorname{Im} \hat{G}_{\epsilon}(\nu)\right] .
\end{aligned}
$$

This expression can be transformed to 


$$
\begin{aligned}
I(\epsilon) & =\frac{1}{\pi} \int_{-\infty}^{\infty} d \nu f(\nu) \operatorname{Tr}\left[2 \sigma_{x} \operatorname{Im} \hat{G}_{\epsilon}(\nu) \sigma_{x} \operatorname{Re} \hat{G}_{\epsilon}(\nu)\right] \\
& =\frac{\operatorname{Tr}}{\pi} \int_{-\infty}^{\infty} d \nu f(\nu) \operatorname{Im}\left[\sigma_{x} \hat{G}_{\epsilon}(\nu) \sigma_{x} \hat{G}_{\epsilon}(\nu)\right] \\
& =\frac{\operatorname{Tr}}{\pi} \int_{-\infty}^{\infty} d \nu f(\nu) \operatorname{Im}\left[\sigma_{x} \frac{d \hat{G}_{\epsilon}(\nu)}{d \epsilon}\right] .
\end{aligned}
$$

The latter equality is obtained using the fact that $d\left(\hat{G}_{\epsilon}^{-1} \hat{G}_{\epsilon}\right) / d \epsilon=d(1) / d \epsilon=0$ and observing that from Eq. (9), one has $d \hat{G}_{\epsilon}^{-1} / d \epsilon=-\sigma_{x}$. Going back to Eq. (C1) and integrating by parts yield

$$
\int_{0}^{\infty} \sigma(\omega)=\sigma_{0} \int_{R B Z} d \epsilon \frac{d(N \phi)}{d \epsilon} \int d \nu f(\nu) 2 \rho_{\epsilon}^{A B}(\nu) .
$$

With the present choice of the current vertex $\phi(\epsilon)$ for the Bethe lattice, one has $\frac{d N \phi}{d \epsilon}=-\epsilon N(\epsilon)$ so that the above expression reduces to

$$
\int_{0}^{\infty} \sigma(\omega)=-\frac{\sigma_{0}}{2} K
$$

where $K$ is the total kinetic energy defined as

$$
K=\int_{\mathrm{RBZ}} d \epsilon N(\epsilon) \epsilon \int d \nu f(\nu) 2 \rho_{\epsilon}^{A B}(\nu) .
$$

Introducing the following spectral density, $\bar{\rho}_{\epsilon_{k}}(\nu)=$ $-\operatorname{Im}\left[\sqrt{z_{A} z_{B}}-\epsilon_{k}\right]^{-1} / \pi$, with $z_{A}$ and $z_{B}$ defined after Eq. (9), and the corresponding momentum distribution function,

$$
n_{\epsilon_{k}}=\int d \nu f(\nu) \bar{\rho}_{\epsilon_{k}}(\nu)
$$

which is the appropriate generalization of the usual $n_{k}$ to the broken-symmetry phase, the total kinetic energy Eq. (C5) can be rewritten as

$$
K=2 \int_{\mathrm{RBZ}} d \epsilon N(\epsilon) \epsilon n_{\epsilon}=2 \sum_{k \in \mathrm{RBZ}} \epsilon_{k} n_{\epsilon_{k}} .
$$

*Present address: ICMM-CSIC, Madrid, Spain.

${ }^{1}$ G. Grüner, Density Waves in Solids (Addison-Wesley, Reading, MA, 1994).

${ }^{2}$ D. S. Dessau, T. Saitoh, C. H. Park, Z. X. Shen, P. Villella, N. Hamada, Y. Moritomo, and Y. Tokura, Phys. Rev. Lett. 81, 192 (1998).

${ }^{3}$ C. P. Adams, J. W. Lynn, Y. M. Mukovskii, A. A. Arsenov, and D. A. Shulyatev, Phys. Rev. Lett. 85, 3954 (2000).

${ }^{4}$ L. Vasiliu-Doloc, S. Rosenkranz, R. Osborn, S. K. Sinha, J. W. Lynn, J. Mesot, O. H. Seeck, G. Preosti, A. J. Fedro, and J. F. Mitchell, Phys. Rev. Lett. 83, 4393 (1999).

${ }^{5}$ T. Katsufuji, T. Tanabe, T. Ishikawa, Y. Fukuda, T. Arima, and Y. Tokura, Phys. Rev. B 54, R14230 (1996).

${ }^{6}$ P. Calvani, A. Paolone, P. Dore, S. Lupi, P. Maselli, P. G. Medaglia, and S.-W. Cheong, Phys. Rev. B 54, R9592 (1996).

${ }^{7}$ C. Bernhard, A. V. Boris, N. N. Kovaleva, G. Khaliullin, A. V. Pimenov, Li Yu, D. P. Chen, C. T. Lin, and B. Keimer, Phys. Rev. Lett. 93, 167003 (2004).

${ }^{8}$ N. L. Wang, Dong Wu, G. Li, X. H. Chen, C. H. Wang, and X. G. Luo, Phys. Rev. Lett. 93, 147403 (2004).

${ }^{9}$ L. Degiorgi, P. Wachter, and D. Ihle, Phys. Rev. B 35, 9259 (1987).

${ }^{10}$ S. K. Park, T. Ishikawa, and Y. Tokura, Phys. Rev. B 58, 3717 (1998).

${ }^{11}$ D. Schrupp, M. Sing, M. Tsunekawa, H. Fujuwara, S. Kasai, A. Sekiyama, S. Suga, T. Muro, V. A. M. Brabers, and R. Claessen, Europhys. Lett. 70, 789 (2005).

${ }^{12}$ C. Presura, M. Popinciuc, P. H. M. van Loosdrecht, D. van der Marel, M. Mostovoy, T. Yamauchi, and Y. Ueda, Phys. Rev. Lett. 90, 026402 (2003).

${ }^{13}$ L. Baldassarre, A. Perucchi, E. Arcangeletti, D. Nicoletti, D. Di Castro, P. Postorino, V. A. Sidorov, and S. Lupi, Phys. Rev. B 75, 245108 (2007).

${ }^{14}$ J. P. Attfield, A. M. T. Bell, L. M. Rodriguez-Martinez, J. M.
Greneche, R. J. Cernik, J. F. Clarke, and D. A. Perkins, Nature (London) 396, 655 (1998).

${ }^{15}$ L. Perfetti, H. Berger, A. Reginelli, L. Degiorgi, H. Hochst, J. Voit, G. Margaritondo, and M. Grioni, Phys. Rev. Lett. 87, 216404 (2001).

${ }^{16}$ L. Perfetti, S. Mitrovic, G. Margaritondo, M. Grioni, L. Forro, L. Degiorgi, and H. Hochst, Phys. Rev. B 66, 075107 (2002).

${ }^{17}$ T. Vuletic, B. Korin-Hamzic, T. Ivek, S. Tomic, B. P. Gorshunov, M. Dressel, and J. Akimitsu, Phys. Rep. 428, 169 (2006).

${ }^{18}$ M. Dressel and N. Drichko, Chem. Rev. (Washington, D.C.) 104, 5689 (2004).

${ }^{19}$ R. Pietig, R. Bulla, and S. Blawid, Phys. Rev. Lett. 82, 4046 (1999).

${ }^{20}$ N.-H. Tong, S.-Q. Shen, and R. Bulla, Phys. Rev. B 70, 085118 (2004).

${ }^{21}$ M. Daghofer, R. M. Noack, and P. Horsch, arXiv:0711.1990 (unpublished).

${ }^{22}$ F. Walz, J. Phys.: Condens. Matter 14, R285 (2002).

${ }^{23}$ S. Ciuchi and F. de Pasquale, Phys. Rev. B 59, 5431 (1999).

${ }^{24}$ S. R. Hassan and H. R. Krishnamurthy, Phys. Rev. B 76, 205109 (2007).

${ }^{25}$ O. P. Matveev, A. M. Shvaika, and J. K. Freericks, Phys. Rev. B 77, 035102 (2008).

${ }^{26}$ C. A. Perroni, V. Cataudella, G. De Filippis, G. Iadonisi, V. M. Ramaglia, and F. Ventriglia, Phys. Rev. B 67, 094302 (2003).

${ }^{27} \mathrm{~A}$ BCS-like prediction $T_{c} \propto \exp (-$ const $/ \lambda)$, valid in the limit $\lambda$ $\rightarrow 0$, actually overestimates the critical temperature at any finite $\lambda$ since it neglects the possible fluctuations of the order parameter (see Refs. 23 and 30).

${ }^{28}$ S. Fratini and G. Rastelli, Phys. Rev. B 75, 195103 (2007).

${ }^{29}$ S. Blawid and A. J. Millis, Phys. Rev. B 63, 115114 (2001).

${ }^{30}$ S. Blawid and A. Millis, Phys. Rev. B 62, 2424 (2000).

${ }^{31}$ A. Georges et al., Rev. Mod. Phys. 68, 13 (1996).

${ }^{32}$ A. Khurana, Phys. Rev. Lett. 64, 1990 (1990). 
${ }^{33}$ H. Schweitzer and G. Czycholl, Phys. Rev. Lett. 67, 3724 (1991).

${ }^{34}$ Th. Pruschke, D. L. Cox, and M. Jarrell, Phys. Rev. B 47, 3553 (1993).

${ }^{35}$ W. Chung and J. K. Freericks, Phys. Rev. B 57, 11955 (1998).

${ }^{36}$ A. Chattopadhyay, A. J. Millis, and S. Das Sarma, Phys. Rev. B 61, 10738 (2000).

${ }^{37}$ S. Fratini and S. Ciuchi, Phys. Rev. B 74, 075101 (2006).

${ }^{38}$ S. Fratini and S. Ciuchi, Phys. Rev. B 72, 235107 (2005).

${ }^{39}$ This estimate is valid outside the polaronic crossover region. Approaching the polaron crossover coupling both from above and below the $\mathrm{CO}$ temperature, the local displacements strongly deviate from the Gaussian behavior.

${ }^{40}$ A. J. Millis, R. Mueller, and B. I. Shraiman, Phys. Rev. B 54, 5389 (1996).

${ }^{41}$ F. Marsiglio, Phys. Rev. B 73, 064507 (2006).

${ }^{42}$ D. Ihle, Z. Phys. B: Condens. Matter 58, 91 (1985).

${ }^{43}$ S. Fratini and S. Ciuchi, Phys. Rev. Lett. 91, 256403 (2003).

${ }^{44}$ D. Ihle and B. Lorentz, J. Phys. C 18, L647 (1985).

${ }^{45}$ D. Ihle and B. Lorentz, J. Phys. C 19, 5239 (1986).

${ }^{46}$ H. G. Reik, Solid State Commun. 1, 67 (1963).

${ }^{47}$ N. Blümer, Ph.D. thesis, University of Augsburg, 2003. 